\title{
Study of Single-supplier Multi-retailer Centralized Supply Chain with Stochastic Discrete Demand
}

\author{
Chunnian Wei \\ School of Management \\ Shanghai University \\ Shanghai, 200444, China \\ Yihua Wei", PhD \\ School of Management \\ Shanghai University \\ Shanghai, 200444, China
}

\begin{abstract}
This paper studies a centralized supply chain model with one supplier and multiple retailers, in which demand is discrete and stochastic. According to whether the supply quantity can be control or not, and if the supply quantity can be control, the supply capacity is infinite or finite? Three models are constructed respectively: I. Fixed supply quantity; II. Controllable supply quantity with infinite supply capacity; III. Controllable supply quantity with infinite supply capacity. For Model I and III, some properties of the optimal solution are proved, and the model is simplified according to these properties. On this basis, the corresponding Fast Fourier Transform method is constructed to obtain the optimal solution. For model II, the analytical expression of the optimal solution is obtained. Finally, for each model, an numerical example is used to verify the results.
\end{abstract}

Keywords: Centralized Supply Chain; Multi-Retailer; Discrete Stochastic Demand; Fast Fourier Transform

\section{Introduction}

At present, there are many papers on supply chain research, but as Hoque (2011) pointed out, most of the studies consider the situation of a single retailer, while few studies on multi-retailers.

Glock and Kim (2015) studied the integration of suppliers with one of the retailers in a single supplier multi-retailer supply chain. After integration, suppliers supply products to the market through integrated sales channels and other retailers respectively. Chen and Chang (2010) studies the jointly determining of perishable goods supply chain under the conditions of channel coordination, system members jointly determine the optimal retail price, replenishment cycle and delivery quantity. Monthatipkul and Yenradee (2007) studies the determination of the safe inventory level and its location of a single product in a supply chain consisting of a centralized warehouse and multiple retailers under demand uncertainty. Darwish and Odah (2010) establishes the supply chain model of single supplier and multiple retailers under VMI mode. The VMI contract agreement between suppliers and retailers is explicitly included in the model. By choosing a higher penalty cost, the model can easily depict the constraints of the supply chain. Mateen and Chatterjee (2015) establishes analysis models of various methods for VMI. Through these methods, a single supplier-multiple retailer system can coordinate through VMI. Su and Geunes (2013) studies a two-stage supply chain in which the supplier serves a group of retailers in the retail chain. Under the uncertainty of retailers' demand, the supplier must set a price discount for each period within a limited planning period.

All the above studies on multi-retailer supply chain consider continuous demand. However, there are few studies on multi-retailer supply chain consider with discrete stochastic demand. In this paper, we will study the centralized supply chain model of multiple retailers under discrete stochastic demand, three cases will be discussed respectively: I. Fixed supply quantity; II. Decision-making of supply quantity with infinite supply capacity; III. Decision-making of supply quantity with finite supply capacity.

\section{Model and Notation}

Consider a centralized supply chain with one supplier and $m$ retailers.

$Q$ : supply quantity of the supplier;

$Q_{j}$ : the quantity of products allocated to retailer $j(j=1,2 \ldots, m)$; 
$\operatorname{Pr}_{j}$ : retail price of retailer $j$;

$v$ : the unit salvage value of the unsold products;

$c$ : the unit supply cost of the supplier, $P r_{j}>c>v$;

$D_{j}$ : the stochastic demand faced by retailer $j$;

$p_{j k}$ : the probability that retailer $j$ 's demand equals to $k, k=1,2, \ldots \ldots$

The expected sales quantity of retailer $j$ is $\operatorname{Emin}\left(Q_{j}, D_{j}\right)$, and the expected unsold quantity of retailer $j$ is $Q_{j}-\operatorname{Emin}\left(Q_{j}, D_{j}\right)$. The unsold quantity of the supplier is $Q-\sum_{j=1}^{m} Q_{j}$. And then the expected total profit of the supply chain is:

$$
\begin{aligned}
\pi^{C}\left(Q, Q_{1}, Q_{2}, \ldots, Q_{m}\right) & =P r_{j} \cdot \sum_{j=1}^{m} \operatorname{Emin}\left(Q_{j}, D_{j}\right)+v \cdot\left[\sum_{j=1}^{m}\left(Q_{j}-\mathrm{E} \min \left(Q_{j}, D_{j}\right)\right)+Q-\sum_{j=1}^{m} Q_{j}\right]-c \cdot Q \\
& =(v-c) Q+\sum_{j=1}^{m}\left[\left(P r_{j}-v\right) \cdot \operatorname{Emin}\left(Q_{j}, D_{j}\right)\right]
\end{aligned}
$$

We consider two cases, depending on whether the supply quantity $Q$ is controllable or not. For case of controllable supply quantity, we further consider whether the supply capacity of the supplier is finite or not.

\section{Uncontrollable Supply Quantity: Model I}

Now, the supply quantity $Q$ is fixed. We only need to decision the quantity of products allocated to each retailer, $Q_{j}$, to maximize the expect profit of the supply chain. We have the follow model:

$$
\begin{gathered}
\max _{Q_{j}} \pi^{C}\left(Q, Q_{1}, Q_{2}, \ldots, Q_{m}\right)=(v-c) Q+\sum_{j=1}^{m}\left[\left(\operatorname{Pr}_{j}-v\right) \cdot \operatorname{Emin}\left(Q_{j}, D_{j}\right)\right] \\
\text { s.t. } \quad \sum_{j=1}^{m} Q_{j} \leq Q \\
Q_{j} \in \mathrm{Z}^{+}, \quad j=1,2, \ldots, m
\end{gathered}
$$

where $\mathrm{Z}^{+}$represents the set of nonnegative integers. Denote the optimal solution of this model by $\left(Q_{1}^{*}, Q_{2}^{*}, \ldots, Q_{m}^{*}\right)$.

This is a stochastic non-linear integer programming model, which can be solved directly by branching and bounding methods, but the solution will be very cumbersome. For this kind of model, it is usually transformed into a dynamic programming model, which is much simpler to solve.

\subsection{Dynamic Programming Model}

Constructing the follow optimal value function

$$
\begin{gathered}
V_{k}(q)=(v-c) q+\max _{\left(Q_{k}, \ldots, Q_{m}\right)} \sum_{j=k}^{m}\left[\left(P r_{j}-v\right) \cdot \operatorname{Emin}\left(Q_{j}, D_{j}\right)\right] \\
\text { s.t. } \quad \sum_{j=k}^{m} Q_{j} \leq Q_{k}^{\prime} \\
Q_{j} \in \mathrm{Z}^{+}, j=k, \ldots, m
\end{gathered}
$$

Obviously, $V_{1}(Q)=\max \pi^{C}\left(Q, Q_{1}, Q_{2}, \ldots, Q_{m}\right)$ 。

Using the method of stochastic dynamic programming, it can get the optimal value function $v_{k}(q) \quad(k=1,2, \ldots, m)$ satisfies the following Bellman Equation:

$$
V_{k}(q)=\max _{Q_{k} \in \mathbb{Z}^{+}}\left\{\left(P r_{k}-v\right) \cdot \operatorname{Emin}\left(Q_{k}, D_{k}\right)+V_{k+1}\left(q-Q_{k}\right)\right\}
$$

The computational complexity of this dynamic programming approach is of the order of $O\left(m Q^{2}\right)$, it is much simpler than the direct solution model (2), but when the supply quantity $Q$ and the number of retailers $m$ is larger, the computational complexity will still increase dramatically. We need to further construct new methods to reduce the computational complexity. We use the following fast Fourier transform method (see Golub and Van Loan 1996).

\subsection{Fast Fourier Transform Method}

For convenience, We introduce the function $F_{j}\left(Q_{j}\right)=\mathrm{E} \min \left(Q_{j}, D_{j}\right)$, which has the following property: 
Theorem 1. 1) $F_{j}\left(Q_{j}\right)=\sum_{t=1}^{Q_{j}} \mathrm{P}\left(D_{j} \geq t\right)$;

2) $F_{j}\left(Q_{j}\right)$ is an increasing and concave function.

Proof. 1) We have

$$
\begin{aligned}
& F_{j}\left(Q_{j}\right)=\operatorname{Emin}\left(Q_{j}, D_{j}\right)=\sum_{i=1}^{Q_{j}} i \cdot p_{j i}+Q_{j} \sum_{i=Q_{j}+1}^{\infty} p_{j i} \\
& =\sum_{i=1}^{Q_{j}} \sum_{t=i}^{Q_{j}} p_{j t}+Q_{j} \sum_{i=Q_{j}+1}^{\infty} p_{j i}=\sum_{i=1}^{Q_{j}}\left\{\sum_{t=i}^{Q_{j}} p_{j t}+\sum_{t=Q_{j}+1}^{\infty} p_{j t}\right\} \\
& =\sum_{i=1}^{Q_{j}} \sum_{t=i}^{\infty} p_{j t}=\sum_{i=1}^{Q_{j}} \mathrm{P}\left\{D_{j} \geq i\right\}
\end{aligned}
$$

2) calculate first-order and second-order difference for $F_{j}\left(Q_{j}\right)$ respectively, we have

$$
\begin{gathered}
\Delta F_{j}\left(Q_{j}\right)=F_{j}\left(Q_{j}\right)-F_{j}\left(Q_{j}-1\right)=\mathrm{P}\left\{D_{j} \geq Q_{j}\right\} \geq 0 \\
\Delta^{2} F_{j}\left(Q_{j}\right)=\Delta F_{j}\left(Q_{j}\right)-\Delta F_{j}\left(Q_{j}-1\right)=\mathrm{P}\left\{D_{j} \geq Q_{j}\right\}-\mathrm{P}\left\{D_{j} \geq Q_{j}-1\right\}=-p_{j, Q_{j}-1} \leq 0
\end{gathered}
$$

Therefore, $F_{j}\left(Q_{j}\right)$ is an increasing and concave function.

By use of function $F_{j}\left(Q_{j}\right)$, we can rewrite Model (2) as:

$$
\begin{gathered}
\max _{Q_{j}} \pi^{C}\left(Q, Q_{1}, Q_{2}, \ldots, Q_{m}\right)=(v-c) Q+\sum_{j=1}^{m}\left[\left(P r_{j}-v\right) \cdot F\left(Q_{j}\right)\right] \\
\text { s.t. } \quad \sum_{j=1}^{m} Q_{j} \leq Q \\
Q_{j} \in \mathrm{Z}^{+}, \quad j=1,2, \ldots, m
\end{gathered}
$$

Let $\alpha_{j Q_{j}}$ denote the marginal profit increased by increase the allocated quantity to retailers $j$ from $Q_{j}-1$ to $Q_{j}$, we have

$$
\alpha_{j Q_{j}}=\pi^{C}\left(Q, Q_{1}, \ldots, Q_{j}, \ldots, Q_{m}\right)-\pi^{C}\left(Q, Q_{1}, \ldots, Q_{j}-1, \ldots, Q_{m}\right)=\left(P r_{j}-v\right) \Delta F_{j}\left(Q_{j}\right)
$$

Theorem 2. Marginal Profit $\alpha_{j Q_{j}}$ is more than 0 and increasing in $Q_{j}$.

Proof. Can be easy obtained from Theorem 1.

Introduce the following $m \times Q$ marginal profit matrix:

$$
\left[\begin{array}{cccc}
\alpha_{11} & \alpha_{12} & \cdots & \alpha_{1 Q} \\
\alpha_{21} & \alpha_{22} & \cdots & \alpha_{2 Q} \\
\cdots & \vdots & \cdots & \vdots \\
\alpha_{m 1} & \alpha_{m 2} & \cdots & \alpha_{m Q}
\end{array}\right]
$$

It can be easily follow from Theorem 2, the marginal profits in each row $j$ are in descending order; i.e., $\alpha_{j 1} \geq \alpha_{j 2} \geq \cdots \geq \alpha_{j Q}$.

By using the fast Fourier transform method, the optimal solution of model (5) is completely determined by the first $Q$ maximum value in the matrix. The sum of these $Q$ values can obtain the maximum value of the objective function. In those $Q$ value, the number of times the subscript $j$ appears is equal to $Q_{j}^{*}$. And because each row of the matrix decreases monotonously from left to right, when choosing the maximum $Q$ value, each time, only the left-most unselected values in each row need to be compared to obtain the corresponding maximum values. Therefore, only $m$ values need to be compared each time, and the total number of comparisons is $Q$ times. At this time, the computational complexity is reduced to the order of $O(m Q)$.

Next two sections consider that the supplier's supply $Q$ can be controlled. There are two cases to discuss: a) the supplier has a limited supply capacity $C$, that is to say, the supplier's supply quantity can't exceed $C$; b) the supplier's supply capacity is infinite, and the supply decision-making is not limited. 
We first discuss the case of infinite supply capacity.

\section{Controllable Supply Quantity with Infinite Capacity: Model II}

On the basis of model (2), by adding the decision-making of supply quantity $Q$, we can get the expected profit maximization model for this case as follows:

$$
\begin{gathered}
\max _{Q, Q_{j}} \pi^{C}\left(Q, Q_{1}, Q_{2}, \ldots, Q_{m}\right)=(v-c) Q+\sum_{j=1}^{m}\left[\left(P r_{j}-v\right) \cdot \operatorname{Emin}\left(Q_{j}, D_{j}\right)\right] \\
\text { s.t. } \quad \sum_{j=1}^{m} Q_{j} \leq Q \\
Q, Q_{j} \in \mathrm{Z}^{+}, j=1,2, \ldots, m
\end{gathered}
$$

Denote the optimal solution of this model by $\left(Q^{a}, Q_{1}^{a}, Q_{2}^{a}, \ldots, Q_{m}^{a}\right)$.

Theorem 3 If and only if $\sum_{j=1}^{m} Q_{j}=Q$, i.e. when retailers' total allocation quantity is exactly equal to the supplier's supply volume, Model (8) obtains the optimal solution.

Proof. By reverse. Assume the optimal solution of Model (8), $\left(Q^{a}, Q_{1}^{a}, Q_{2}^{a}, \ldots, Q_{m}^{a}\right)$, makes that $\sum_{j=1}^{m} Q_{j}^{a}<Q^{a}$, then

$$
\begin{aligned}
\pi^{c}\left(Q^{a}, Q_{1}^{a}, Q_{2}^{a}, \ldots, Q_{m}^{a}\right) & =\pi^{c}\left(\sum_{j=1}^{m} Q_{j}^{a}, Q_{1}^{a}, Q_{2}^{a}, \ldots, Q_{m}^{a}\right)+(v-c)\left(Q^{a}-\sum_{j=1}^{m} Q_{j}^{a}\right) \\
& <\pi^{c}\left(\sum_{j=1}^{m} Q_{j}^{a}, Q_{1}^{a}, Q_{2}^{a}, \ldots, Q_{m}^{a}\right)
\end{aligned}
$$

this contradicts the definition of the optimal solution $\left(Q^{a}, Q_{1}^{a}, Q_{2}^{a}, \ldots, Q_{m}^{a}\right)$, and implies the supposition made in the beginning of the proof is false. This completes the proof.

By use of Theorem 3, we can replace the decision variable $Q$ with $\sum_{j=1}^{m} Q_{j}$, reduce one decision variable and one constraints, and simplify Model (8) to:

$$
\begin{aligned}
\Pi^{C}=\max _{Q_{j} \in \mathbb{Z}^{+}} \pi^{c}\left(\sum_{j=1}^{m} Q_{j}^{a}, Q_{1}^{a}, Q_{2}^{a}, \ldots, Q_{m}^{a}\right) & =(v-c) \sum_{j=1}^{m} Q_{j}+\sum_{j=1}^{m}\left[\left(\operatorname{Pr}_{j}-v\right) \cdot \operatorname{Emin}\left(Q_{j}, D_{j}\right)\right] \\
& =\sum_{j=1}^{m}\left[\left(\operatorname{Pr}_{j}-v\right) \cdot \operatorname{Emin}\left(Q_{j}, D_{j}\right)+(v-c) Q_{j}\right]
\end{aligned}
$$

Because the objective function of model (9) can be decomposed into separate m parts. Therefore, each part can be maximized separately, i.e.

$$
\Pi_{j}^{C}:=\max _{Q_{j} \in \mathbb{Z}^{+}} \Pi_{j}^{C}\left(Q_{j}\right)=\left(\operatorname{Pr}_{j}-v\right) \cdot \operatorname{Emin}\left(Q_{j}, D_{j}\right)+(v-c) Q_{j}
$$

The maximum expected profit of the whole supply chain equals the sum of the maximum expected profit of each part, i.e. $\Pi^{C}=\sum_{j=1}^{m} \Pi_{j}^{C}$.

The first-order difference and the second-order difference of Profit function $\Pi_{j}^{C}\left(Q_{j}\right)$ are respectively as follows:

$$
\begin{gathered}
\Delta \Pi_{j}^{C}\left(Q_{j}\right)=\Pi_{j}^{C}\left(Q_{j}\right)-\Pi_{j}^{C}\left(Q_{j}-1\right)=\left(P r_{j}-v\right) \cdot\left[F_{j}(n)-F_{j}(n-1)\right]+(v-c) \\
=\left(P r_{j}-v\right) \cdot \mathrm{P}\left\{D_{j} \geq n\right\}+(v-c) \\
\Delta^{2} \Pi_{j}^{C}\left(Q_{j}\right)=\Delta \Pi_{j}^{C}\left(Q_{j}\right)-\Delta \Pi_{j}^{C}\left(Q_{j}-1\right)=\left(P r_{j}-v\right) \cdot\left[\mathrm{P}\left\{D_{j} \geq n\right\}-\mathrm{P}\left\{D_{j} \geq n-1\right\}\right]=-p_{j, n-1} \leq 0
\end{gathered}
$$

Hence, property of $\Pi_{j}^{C}\left(Q_{j}\right)$ is given by the follows theorem:

Theorem $4 \Pi_{j}^{C}\left(Q_{j}\right)$ is a concave function of $Q_{j}$, i.e. $\Delta \Pi_{j}^{C}\left(Q_{j}\right)$ is increasing in $Q_{j}$.

Let $L_{j}^{a}=\left\{l \in Z^{+}, \Delta \Pi_{j}^{C}(l) \geq 0\right\}$ denote the sets of allocation quantity which makes $\Delta \Pi_{j}^{C}\left(Q_{j}\right)$ non-negative, denotes the maximum value of the set be $l_{j}^{a}$. Follow from Theorem 4, we can obtain: if $Q_{j} \leq l_{j}^{a}$, then $\Pi_{j}^{c}\left(Q_{j}\right)$ is monotonic increasing; else, $\Pi_{j}^{C}\left(Q_{j}\right)$ is monotonic decreasing. 
Hence, $l_{j}^{a}$ is the maximum point of Revenue Function $\Pi_{j}^{C}\left(Q_{j}\right)$. And then, the optimal solution of Model (9) will be

$$
Q_{j}^{a}=l_{j}^{a}=\max _{l}\left\{l \in Z^{+}, \Delta \Pi_{j}^{C}(l) \geq 0\right\} \quad j=1, \ldots, m
$$

\section{Controllable Supply Quantity with Finite Capacity: Model III}

At this time, the supplier's supply quantity $Q$ can't exceed the supply capacity $C$. On the basis of model (9), a constraint condition on supply quantity is added, and the model becomes

$$
\begin{gathered}
\max \pi^{C}\left(\sum_{j=1}^{m} Q_{j}, Q_{1}, Q_{2}, \ldots, Q_{m}\right)=\sum_{j=1}^{m}\left[\left(P r_{j}-v\right) \cdot \operatorname{Emin}\left(Q_{j}, D_{j}\right)+(v-c) Q_{j}\right] \\
\text { s.t. } \quad \sum_{j=1}^{m} Q_{j} \leq C \\
Q_{j} \in \mathrm{Z}^{+}, \quad j=1,2, \ldots, m
\end{gathered}
$$

Denote the optimal solution of this model by $\left(Q^{A}, Q_{1}^{A}, Q_{2}^{A}, \ldots, Q_{m}^{A}\right)$.

Let $A_{j Q_{j}}$ denote the marginal profit comes from increase the allocated quantity to retailers $j$ from $Q_{j}-1$ to $Q_{j}$, we have

$$
\begin{aligned}
A_{j Q_{j}} & =\pi^{c}\left(\sum_{j=1}^{m} Q_{j}, Q_{1}, \ldots, Q_{j}, \ldots, Q_{m}\right)-\pi^{c}\left(\sum_{j=1}^{m} Q_{j}-1, Q_{1}, \ldots, Q_{j}-1, \ldots, Q_{m}\right) \\
& =\left(P r_{1}-v\right) F_{j}\left(Q_{j}\right)-\left(P r_{1}-v\right) F_{j}\left(Q_{j}-1\right)+(v-c) \\
& =\left(\operatorname{Pr}_{1}-v\right) \mathrm{P}\left\{D_{j} \geq Q_{j}\right\}+(v-c) \\
& =\Delta \Pi_{j}^{C}\left(Q_{j}\right)
\end{aligned}
$$

The following $m \times C$ marginal profit matrix is introduced by using fast Fourier transform method:

$$
\left[\begin{array}{ccll}
A_{11} & A_{12} & \cdots & A_{1 S C} \\
A_{21} & A_{22} & \cdots & A_{2 S C} \\
\cdots & \vdots & \cdots & \vdots \\
A_{m 1} & A_{m 2} & \cdots & A_{m S C}
\end{array}\right]
$$

If the number of positive values in matrix (15) is greater than $C$, then the supply quantity is $C$, and maximum $C$ values in the matrix corresponds to the optimal allocation rule at this time; else, the optimal solution is the same as formula (12).

\section{Numerical Example}

Consider a supply chain with one supplier and four retailers. The supplier's unit supply cost is $\$ 1800$, and the unit salvage value of unsold items is $\$ 300$. Retail price of retailer 1 is $\$ 3000$, and demand of retailer 1 follows a Poisson distribution with parameter 7; retail price of retailer 2 is $\$ 2300$, and demand of retailer 2 follows a Poisson distribution with parameter 7.9; retail price of retailer 3 is $\$ 2500$, and demand of retailer 3 follows a Poisson distribution with parameter 8.1; retail price of retailer 3 is $\$ 3500$, and demand of retailer 4 follows a Poisson distribution with parameter 4.8 .

The initial parameter information is summarized in the following table:

Table 1 Parameter value

\begin{tabular}{|c|c|c|c|c|c|c|c|c|c|c|c|}
\hline Parameter & $m$ & $c$ & $v$ & $P r_{1}$ & $P r_{2}$ & $P r_{3}$ & $P_{4}$ & $\lambda_{1}$ & $\lambda_{2}$ & $\lambda_{3}$ & $\lambda_{4}$ \\
\hline Value & 4 & 1800 & 300 & 3000 & 2300 & 2500 & 3500 & 7.0 & 7.9 & 8.1 & 4.8 \\
\hline
\end{tabular}

\subsection{Uncontrollable Supply Quantity: Model I}

The supply quantity is fixed as 20 items, By introducing related parameters values into matrix (7), we can get the follows: $4 \times 20$ order matrix: 


$\left[\begin{array}{ccccccccc}2697.50 & 2680.26 & 2619.94 & 2479.19 & 2232.88 & 1888.05 & 1485.74 & \cdots & 0.12 \\ 1999.10 & 1993.24 & 1970.10 & 1909.17 & 1788.84 & 1598.70 & 1348.36 & \cdots & 0.43 \\ 2199.09 & 2193.68 & 2171.77 & 2112.62 & 1992.85 & 1798.81 & 1536.87 & \cdots & 0.65 \\ 3173.66 & 3047.26 & 2743.87 & 2258.46 & 1675.97 & 1116.78 & 669.43 & \cdots & 0\end{array}\right]$

We only need to select the maximum 20 values from the above matrix, and then the optimal solution of the model can be obtained. At this time, the optimal allocation quantity of retailer 1 is 6 , the optimal allocation quantity of retailer 2 is 4 , the optimal allocation quantity of retailer 3 is 6 , and the allocation quantity of retailer 4 is 4 . The expected profit of the supply chain is:

$$
\max _{Q_{1}, Q_{2}, Q_{3}, Q_{4}} \pi^{c}\left(Q_{1}, Q_{2}, Q_{3}, Q_{4}\right)=\sum(\text { maximum } 20 \text { values })-(c-v) Q=16161.5
$$

\subsection{Controllable Supply Quantity with Infinite Capacity: Model II}

Introducing related parameters value into formula (11), we have:

$$
\begin{gathered}
\Delta \Pi_{1}^{C}(6)=388.05>0, \Delta \Pi_{1}^{C}(7)=-14.26<0 \\
\Delta \Pi_{2}^{C}(6)=78.70>0, \Delta \Pi_{2}^{C}(7)=-151.64<0 \\
\Delta \Pi_{3}^{C}(7)=36.87>0, \Delta \Pi_{3}^{C}(8)=-226.25<0 \\
\Delta \Pi_{4}^{C}(5)=175.97>0, \Delta \Pi_{4}^{C}(6)=-383.22<0
\end{gathered}
$$

Combination with formula (12), we can get the optimal allocated quantity to each retailer be

$$
Q_{1}^{a}=6, Q_{2}^{a}=6, Q_{1}^{a}=7, Q_{4}^{a}=5
$$

\begin{tabular}{|c|c|c|c|c|c|c|c|c|}
\hline 197.50 & 1180.26 & 1119.94 & 979.19 & 732.88 & 388.05 & -14.26 & -416.57 & .887 \\
\hline 499.10 & 493.24 & 470.10 & 409.17 & 288.84 & 98.70 & -151.64 & -434.17 & 9.57 \\
\hline 699. & 693 & 671.77 & 612.62 & 492.85 & 298.81 & 36.87 & -266.25 & $\cdots-1499.35$ \\
\hline 73.6 & 1547.26 & 1543.87 & 758.46 & 175.97 & -383.22 & -830.57 & -1137.33 & -1500 \\
\hline
\end{tabular}

And the corresponding supplier's optimal supply quantity is 24 items, and the total expected profit of the supply chain is $\$ 16761.91$.

\subsection{Controllable Supply Quantity with Finite Capacity: Model III}

By introducing related parameters values into matrix (15), we can get the follows: $4 \times 20$ order matrix:

There are 24 positive values in the above matrix. That is to say, the supplier's maximal supply quantity is 24 items. When the supplier's supply capacity $C$ is less than 24 items, his supply quantity is $C$; else, his supply quantity is 24 items. Table 2 gives the optimal policy under different supply capabilities.

Table 2 Optimal policy under different supply capabilities

\begin{tabular}{|c|c|c|c|c|}
\hline Supply capacity & Supply quantity & Marginal profit & Total expected profit & Optimal allocated policy \\
\hline 1 & 1 & 1673.66 & 1673.66 & $(0,0,0,1)$ \\
\hline 2 & 2 & 1547.26 & 3220.92 & $(0,0,0,2)$ \\
\hline 3 & 3 & 1243.87 & 4464.79 & $(0,0,0,3)$ \\
\hline 4 & 4 & 1197.5 & 5662.29 & $(2,0,0,3)$ \\
\hline 5 & 5 & 1180.26 & 6842.55 & $(3,0,0,3)$ \\
\hline 6 & 6 & 1119.94 & 7962.49 & $(4,0,0,3)$ \\
\hline 7 & 7 & 979.19 & 8941.68 & $(5,0,0,4)$ \\
\hline 8 & 8 & 758.46 & 9700.14 & $(5,0,1,4)$ \\
\hline 9 & 9 & 732.88 & 10433.02 & $(5,0,2,4)$ \\
\hline 10 & 10 & 699.09 & 11132.11 & $(5,0,3,4)$ \\
\hline 11 & 11 & 693.68 & 11825.79 & $(5,0,4,4)$ \\
\hline 12 & 12 & 671.77 & 12497.56 & $(5,2,4,4)$ \\
\hline 13 & 13 & 612.62 & 13110.18 & \\
\hline 14 & 14 & 499.1 & 13609.28 & 14102.52 \\
\hline 15 & 15 & 493.24 & & \\
\hline
\end{tabular}




\begin{tabular}{|l|l|c|c|c|}
\hline 16 & 16 & 492.85 & 14595.37 & $(5,2,5,4)$ \\
\hline 17 & 17 & 470.1 & 15065.47 & $(5,3,5,4)$ \\
\hline 18 & 18 & 409.17 & 15474.64 & $(5,4,5,4)$ \\
\hline 19 & 19 & 388.05 & 15862.69 & $(6,4,5,4)$ \\
\hline 20 & 20 & 298.81 & 16161.5 & $(6,4,6,4)$ \\
\hline 21 & 21 & 288.84 & 16450.34 & $(6,5,6,5)$ \\
\hline 22 & 22 & 175.97 & 16626.31 & $(6,6,6,5)$ \\
\hline 23 & 23 & 98.7 & 16725.01 & $(6,6,7,5)$ \\
\hline 24 & 24 & 36.87 & 16761.88 & $(6,6,7,5)$ \\
\hline 25 & 24 & 0 & 16761.88 & $22(\mathrm{it}, \mathrm{and}$ \\
\hline
\end{tabular}

For example, if the supplier's supply capacity is 22 items, then his optimal supply capacity is 22 items, and the optimal allocated policy is $(6,5,6,5)$, i.e. the optimal allocation quantity of retailer 1 is 6 , the optimal allocation quantity of retailer 2 is 5 , the optimal allocation quantity of retailer 3 is 6 , and the allocation quantity of retailer 4 is 5 . And the expected profit of the supply chain is $\$ 16626.31$.

\section{Conclusion}

We study a centralized supply chain model of single supplier and multiple retailers under discrete stochastic demand. We discuss two situations according to whether the supplier make decisions on supply quantity. If the supplier make decisions on supply quantity, we also discuss two cases, that is, infinite supply capacity and finite supply capacity. Three models are established for analysis and discussion. For each model, some properties of its optimal solution are proved. According to these properties, the original model is simplified, and an improved algorithm for solving these models is given.

\section{References}

Bertsekas, D. P. 1999. Nonlinear Programming, 2nd ed. Athena Scientific, Belmont, MA.

Chen, T. H., \& Chang, H. M. (2010). Optimal ordering and pricing policies for deteriorating items in one-vendor multi-retailer supply chain[J]. International Journal of Advanced Manufacturing Technology, 49(1-4), 341-355.

Chiou, C. C., Yao, M. J., \& Tsai, J. (2007). A mutually beneficial coordination mechanism for a one-supplier multi-retailers supply chain[J]. International Journal of Production Economics, 108(1-2), 314-328.

Darwish, M. A., \& Odah, O. M. (2010). Vendor managed inventory model for single-vendor multi-retailer supply chains[J]. European Journal of Operational Research, 204(3), 473-484.

Glock, C. H., \& Kim, T. (2015). The effect of forward integration on a single-vendor-multi-retailer supply chain under retailer competition[J]. International Journal of Production Economics, 164, 179-192.

Golub, G. H., C. F. Van Loan. 1996. Matrix Computations, 3rd ed. The Johns Hopkins University Press, Baltimore.

Hoque, M.A. 2011. Generalized single-vendor multi-buyer integrated inventory supply chain models with a better synchronization[J]. International Journal of Production Economics, 131: 463-472.

Mateen, A., \& Chatterjee, A. K. (2015). Vendor managed inventory for single-vendor multi-retailer supply chains[J]. Decision Support Systems, 70, 31-41.

Mateen, A., Chatterjee, A. K., \& Mitra, S. (2015). VMI for single-vendor multi-retailer supply chains under stochastic demand[J]. Computers \& Industrial Engineering, 79, 95-102.

Monthatipkul, C., \& Yenradee, P. (2007). Positioning safety stock in a one-warehouse multi-retailer supply chain controlled by optimal inventory/distribution plan[J]. International Journal of Industrial Engineering-Theory Applications and Practice, 14(2), 169-178.

Monthatipkul, C., \& Yenradee, P. (2008). Inventory/distribution control system in a one-warehouse/multi-retailer supply chain[J]. International Journal of Production Economics, 114(1), 119-133.

Pan F, Nagi R. Robust supply chain design under uncertain demand in agile manufacturing[J]. Computers \& Operations Research, 2010, 37(4): 668-683.

Schonlein, M., Makuschewitz, T., Wirth, F., \& Scholz-Reiter, B. (2013). Measurement and optimization of robust stability of multiclass queueing networks: Applications in dynamic supply chains[J]. European Journal of Operational Research, 229(1), $179-189$.

Su, Y. Q., \& Geunes, J. (2013). Multi-period price promotions in a single-supplier, multi-retailer supply chain under asymmetric demand information[J]. Annals of Operations Research, 211(1), 447-472.

Yang, P. C., Chung, S. L., Wee, H. M., Zahara, E., Peng, C. Y. (2013). Collaboration for a closed-loop deteriorating inventory supply chain with multi-retailer and price-sensitive demand[J]. International Journal of Production Economics, 143(2), 557-566.

Yihua Wei, Chen Xu, Qiying Hu, 2013. Transformation of optimization problems in revenue management, queueing system, and supply chain management[J]. International Journal of Production Economics, 146(2), 588-597.

Ziya,S., Ayhan,H., Foley,R.D., 2004. Relationships among three assumption in revenue management[J]. Operations Research 52, 804-809. 